\section{Comment on "Ear Deformations Give Bats a Physical Mechanism for Fast Adaptation of Ultrasonic Beam Patterns"}

Gao et al. [1] conclude that horseshoe bats could use nonrigid ear deformations to switch between functionally different head-related transfer functions (HRTFs). We argue that, while it is possible that the ear deformations introduce additional directional cues relevant to the bat, the presented evidence is insufficient to support this conclusion. First, the coordinate system of the HRTFs is not referred to a behaviorally relevant coordinate system fixed to the bat's head. Second, as sonar is an active sense, the spatial sensitivity of the system cannot be determined by analyzing the HRTF only [2-4]. Drawing conclusions about the relevance of the observed HRTF changes requires both the emission pattern and the HRTF to be calculated and referred to a behaviorally relevant coordinate system. We understand that the approach taken in this study does not allow relating the pinna centered coordinate system used to a head centered one. Hence, by matching the mainlobes of Gao et al. (Fig. 3 of Ref. [1]) to the data of Grinnell and Schnitzler $[5,6]$ on the hearing sensitivity of Rhinolophus ferrumequinum, we have related the azimuth and elevation angles for the reported sidelobes to the relevant coordinate system as defined in Ref. [6]. This leaves one unspecified rotational degree of freedom, i.e., a rotation around the anteroposterior axis. Based on Figs. (7)-(10) of the Supplemental Material of Ref. [1] and a comparison of the relative positions of the sidelobes and the mainlobes for the upright position of the pinna with those reported by Firzlaff and Schuller [7] for $R$. rouxi, we assume this remaining angle to be zero. Figure 1 (left) reveals that the sidelobes in Fig. 3(c) are located at peripheral positions around $90^{\circ}$ azimuth. Furthermore, taking into account pinna rotations as well [8], the sidelobes will move to even more peripheral positions, i.e., by an additional rotation of $15^{\circ}$ (this effect can be observed in the movie provided as Supplemental Material in Ref. [1]).

While behavioral experiments [9] show that ear movements are functionally relevant, i.e., provide the bat with spatial information, it is not known through what cues this information is conveyed. Simulations and robotic experiments have shown that rotation of the HRTF mainlobe provides spatial cues allowing one to locate targets in the frontal hemisphere [10-12]. However, to determine whether it is this mechanism that underlies the function of the ear movements further experimental evidence on the precise timing, duration, and synchronicity of ear movements and sonar emissions is required. Nevertheless, this raises the possibility for an alternative interpretation of the results reported by Gao et al. [1]; i.e., the observed sidelobe changes are merely a side effect of realizing this mainlobe rotation. Given that a flexible pinna actuated from its base shows both a pinna rotation and deformation component, we interpret the findings by Gao et al. to show that a

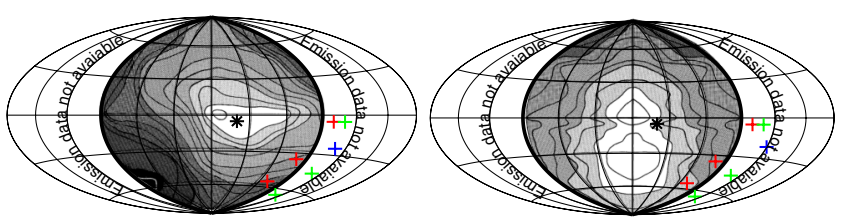

FIG. 1 (color online). Measured spatial sensitivity patterns of $R$. ferrumequinum (no experimental data available beyond frontal hemisphere) $[5,6]$. Contour lines are spaced $3 \mathrm{~dB}$ apart. Left: Hearing pattern. The asterisk indicates the reference point taken as the center of the hearing mainlobe. The colored crosses indicate the positions of the sidelobes for three different pinna positions in Fig. 3(c) in Goa et al. [1]. Red, pinna position 2; green, pinna position 3; blue, pinna position 4. Right: Emission pattern. Markers similar to those in the left panel. The angular interval between the azimuth and elevation parallels is $30 \mathrm{deg}$.

rotating HRTF mainlobe will, in general, be accompanied by changing sidelobes. However, we interpret the peripheral positions of the sidelobes to indicate that the pinna shape and its deformations have evolved to generate sidelobes that do not interfere with the main spatial cues provided by the rotating HRTF mainlobe. Hence, the observed pinna movements, rigid and nonrigid components combined, can be interpreted as an efficient way to generate HRTF changes that are functionally similar to a simple rotation of the HRTF mainlobe.

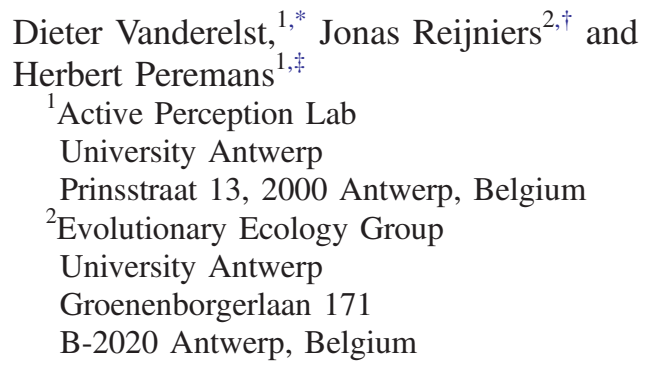

Received 8 June 2012; published

DOI: 10.1103/PhysRevLett.112.079401

PACS numbers:

*dieter.vanderelst@uantwerpen.be †jonas reijniers@uantwerpen.be herbert.peremans@uantwerpen.be

[1] L. Gao, S. Balakrishnan, W. He, Z. Yan, and R. Müller, Phys. Rev. Lett. 107, 214301 (2011).

[2] Z. Fuzessery, D. Hartley, and J. Wenstrup, J. Comp. Physiol. A 170, 57 (1992).

[3] D. Vanderelst, F. De Mey, H. Peremans, I. Geipel, E. Kalko, and U. Firzlaff, PLoS One 5, e11893 (2010).

[4] J. M. Wotton, R. L. Jenison, and D. J. Hartley, J. Acoust. Soc. Am. 101, 1723 (1997).

[5] A. Grinnell and H. Schnitzler, J. Comp. Physiol. A 116, 63 (1977).

[6] H. Schnitzler and A. Grinnell, J. Comp. Physiol. A 116, 51 (1977). 
[7] U. Firzlaff and G. Schuller, Hear. Res. 197, 74 (2004).

[8] J. Pye, M. Flinn, and A. Pye, Nature (London) 196, 1186 (1962).

[9] J. Mogdans, J. Ostwald, and H. Schnitzler, J. Acoust. Soc. Am. 84, 1676 (1988).
[10] V. A. Walker, H. Peremans, and J. C. Hallam, J. Acoust. Soc. Am. 104, 569 (1998).

[11] D. Vanderelst, J. Reijniers, J. Steckel, and H. Peremans, PLoS One 6, e20627 (2011).

[12] J. Carmena and J. Hallam, Robot. Auton. Syst. 46, 247 (2004). 